\title{
ACE for whom? Implications for clinical practice of post-infarct trials
}

Division of

Cardiovascular

Medicine, University

Hospital, Nottingham

J T Walsh

D Gray

A J Cowley

$\mathrm{J} R$ Hampton

Cardiovascular

Statistics Unit, British

Heart Foundation,

Department of

Mathematics,

University of

Nottingham,

Nottingham

N A Keating

Correspondence to:

Dr J TWalsh, Division of

Cardiovascular Medicine,

University Hospital,

Nottingham NG7 2UH.

Accepted for publication 18 October 1994

\begin{abstract}
Objective-To determine how many lives would be saved if patients were routinely treated with ACE inhibitors after myocardial infarction according to the criteria of four recent major clinical trials, and to estimate the costs and benefits of these approaches.

Design-Retrospective survey.

Setting-The Nottingham Health District. Patients-Data from 7855 patients admitted between 1989 and 1990 were combined and the selection criteria of four major clinical trials (AIRE, SAVE, GISSI-3, and ISIS-4) were applied.

Results-Of the patients admitted in Nottingham with confirmed myocardial infarcts 39\% were eligible for AIRE and $8 \%$ for SAVE. In patients with suspected myocardial infarction as defined by the major trials, $60 \%$ would have been eligible for GISSI-3 and $63 \%$ for ISIS-4. Treating appropriate patients in accordance with these trials would have saved 20 (AIRE), 3 (SAVE), 4 (GISSI-3) and 5 (ISIS-4) lives each year in Nottingham at a drug cost of $£ 5400, £ 33791, £ 2730$, and $£ 4116$ per life per year saved respectively. Conclusions-Short-term treatment with ACE inhibition appears to be cheaper but such an approach would save fewer lives. The AIRE study is the most applicable to current clinical practice but ACE inhibitors should be offered routinely to patients satisfying the criteria of any of the four major clinical trials.
\end{abstract}

(Br Heart f 1995;73:470-474)

Keywords: myocardial infarction; angiotensin converting enzyme inhibitors; clinical trials

Several factors influence survival after a myocardial infarction: the most important factor seems to be the severity of left ventricular dysfunction. ${ }^{1}$ The importance of left ventricular volume is now established, with ventricular enlargement identified as a significant risk factor in the development of cardiac failure. ${ }^{2}$ In the Framingham Heart Study the risk of symptomatic heart failure developing in patients with a myocardial infarction was 7 to 10 times that of the normal population. ${ }^{3}$ In view of the appalling prognosis of chronic heart failure, even in those treated with angiotensin converting enzyme (ACE) inhibitors, ${ }^{4}$ much research has been aimed at treatments that might help to prevent the onset of heart failure.

Four large multicentre studies ${ }^{5-8}$ have now reported beneficial effects of ACE inhibitors after a myocardial infarct but their relevance to current clinical practice remains unclear, largely because the populations randomised in these trials were very different owing to variation in inclusion and exclusion criteria. In SAVE, asymptomatic patients were screened for evidence of left ventricular dysfunction ${ }^{5}$; in AIRE, patients with clinical evidence of heart failure were recruited without objective assessment of ventricular function ${ }^{6}$; and the GISSI-3 and ISIS-4 trials included patients with suspected myocardial infarct presenting within 24 hours of the onset of pain. ${ }^{78}$

We wanted to assess the impact for our hospitals, in terms of reduced mortality, of a policy of ACE inhibition as suggested by the major trials and to estimate the costs of such a policy. We applied selection criteria from the four major trials to data from the Nottingham Heart Attack Register, ${ }^{9}$ which has documented all patients brought to the Nottingham hospitals (alive or dead) with suspected myocardial infarction during selected periods since 1973, to determine how applicable these trials were to patients from the Nottingham Health district.

\section{Patients and methods}

The methods of data collection and storage for the Nottingham Heart Attack Register have been described elsewhere. ${ }^{9}$ All patients admitted to the Nottingham hospitals with symptoms suggestive of acute myocardial infarction were identified through the medical records departments, accidents and emergency departments, and the records of the Nottingham Ambulance service. Most patients $(75 \%)$ were admitted to coronary care but data from all admissions were used for analysis. A suspected myocardial infarct was defined as the primary working diagnosis, based upon the presenting history and initial electrocardiogram, as recorded by the admitting physician. A myocardial infarct was confirmed according to the criteria defined in the major trials..$^{5-8}$ Details of management including Killip scores on admission, drugs prescribed while an inpatient and on discharge, the final diagnosis, and the outcome of the 
admission were obtained from the case notes after discharge or death of the patient. For this study we present data from 1989 and 1990, before ACE inhibitors were recommended after myocardial infarction.

Patients admitted with suspected myocardial infarction to the Nottingham hospitals were identified from their entry on the Heart Attack Register so that they matched as closely as possible the characteristics of the patients recruited into the four postinfarction studies. To do this we applied the selection criteria used to derive the population for the AIRE study to the Nottingham patients. Those Nottingham patients who fulfilled all the inclusion and exclusion criteria (and so who would have satisfied the entry requirements for the AIRE study) were used for this analysis.

We then applied the SAVE, GISSI-3, and ISIS-4 selection criteria to identify Nottingham patients who would have met the specific entry requirements for the SAVE, GISSI- 3 and ISIS- 4 studies.

We used the mortality data from these studies to calculate the potential impact on survival after a myocardial infarct for the Nottingham patients. These data were then used to estimate the drug cost per year per life

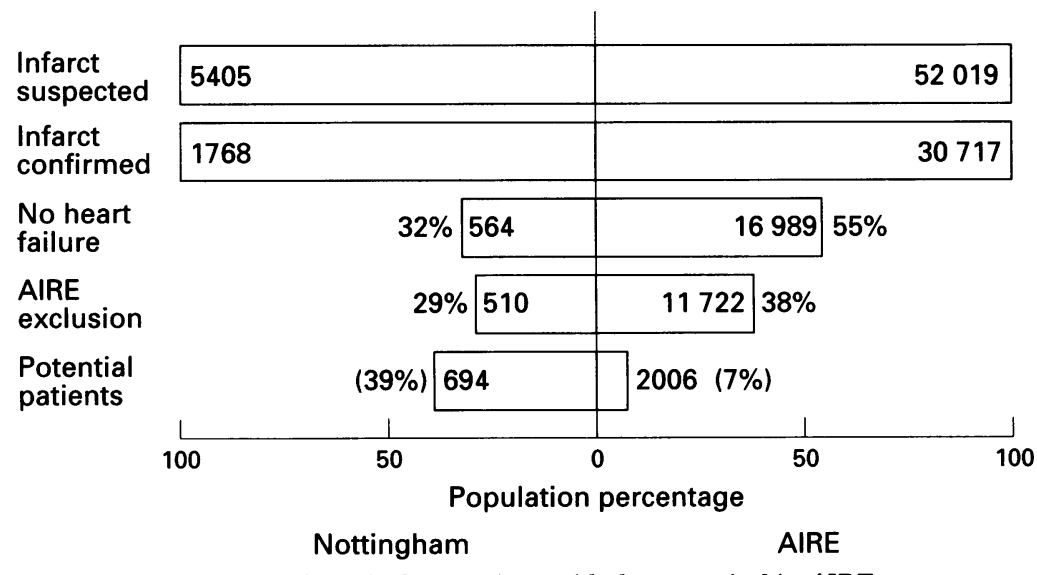

Figure 1 Comparison of Nottingham patients with those recruited in AIRE.

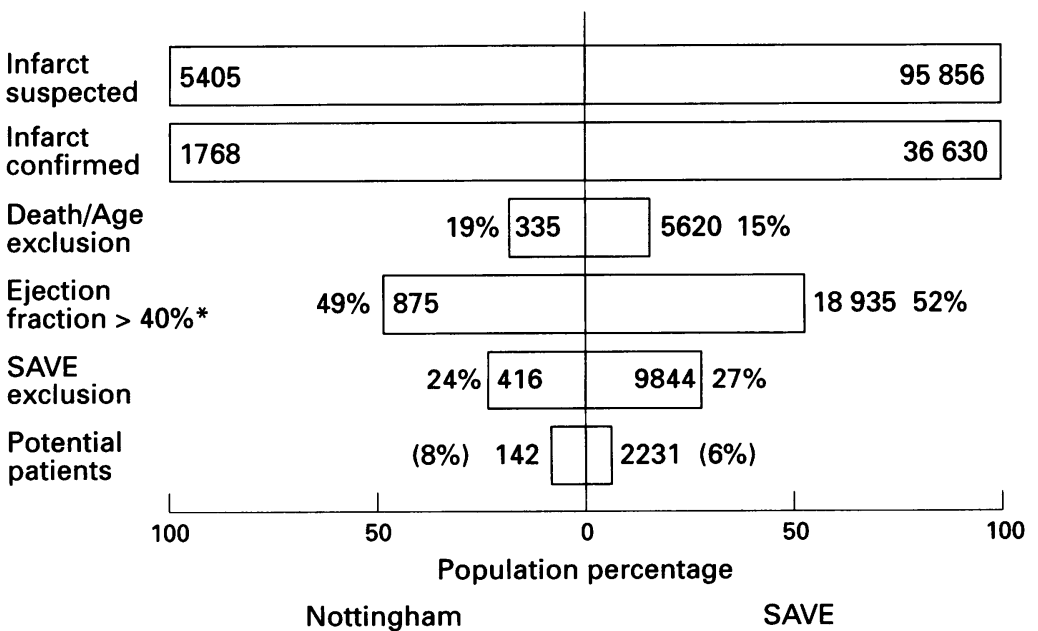

Figure 2 Comparison of Nottingham patients with those recruited in SAVE (*estimated from SAVE data). saved if patients were treated as described in the major trials. Drug costs were obtained from the British National Formulary.

\section{Results}

COMPARISON OF NOTTINGHAM PATIENTS WITH THOSE RECRUITED IN AIRE

AIRE study

Figure 1 shows the patients considered for entry to the AIRE Study and the reasons for excluding patients from the study. More than 52000 were considered; 30717 (59\% of all patients) had a confirmed myocardial infarction and almost 17000 (55\% of those with infarcts) had no signs (even transiently) of heart failure; 11722 (38\% of those with infarcts) were specifically excluded because of unstable angina, severe heart failure, because of intolerance of (or because they were already taking) ACE inhibitors, or because of sustained hypotension. This figure also includes 3323 (11\% of those with infarcts) patients in whom long-term assessment or consent were impracticable. Overall, 2006 (7\% of all those with infarcts) were recruited for the AIRE Study.

\section{Nottingham patients}

Figure 1 also shows that in Nottingham 5405 patients had a suspected myocardial infarction between 1989 and 1990; over 1700 (33\% of all patients) had a confirmed myocardial infarction; of these 564 (32\% of those with infarcts) had no signs, even transiently, of heart failure. $1204 \quad(68 \%$ of those with infarcts) had overt heart failure but 510 $(29 \%)$ of those with infarcts) would have been specifically excluded by the AIRE criteria. This figure does not include patients in whom consent or long-term assessment was impracticable. Overall, 694 (39\% of all those with infarcts) would have met the entry requirements for the AIRE study.

COMPARISON OF NOTTINGHAM PATIENTS WITH THOSE RECRUITED IN SAVE

SAVE study population

Figure 2 shows the patients considered for entry to the SAVE study and the reasons why patients were excluded. Nearly 96000 patients were considered for recruitment: 36630 (38\% of all patients) had an infarct confirmed but 5620 (15\% of infarcts) of these were excluded owing to death within 3 days or age ineligibility; of the $31010(81 \%$ of those with infarcts) remaining 18935 (52\% of those with infarcts) were excluded because they had an ejection fraction $>40 \%$ on a radionuclide scan. A further $9844(27 \%$ of infarcts) were excluded because of preexisting congestive cardiac failure, development of severe heart failure, unstable angina, sustained hypotension, and intolerance of ACE inhibitors. Overall 2321 (6\% of all those with infarcts) patients were randomised into the study.

\section{Nottingham patients}

Figure 2 also shows that of the 5405 patients 


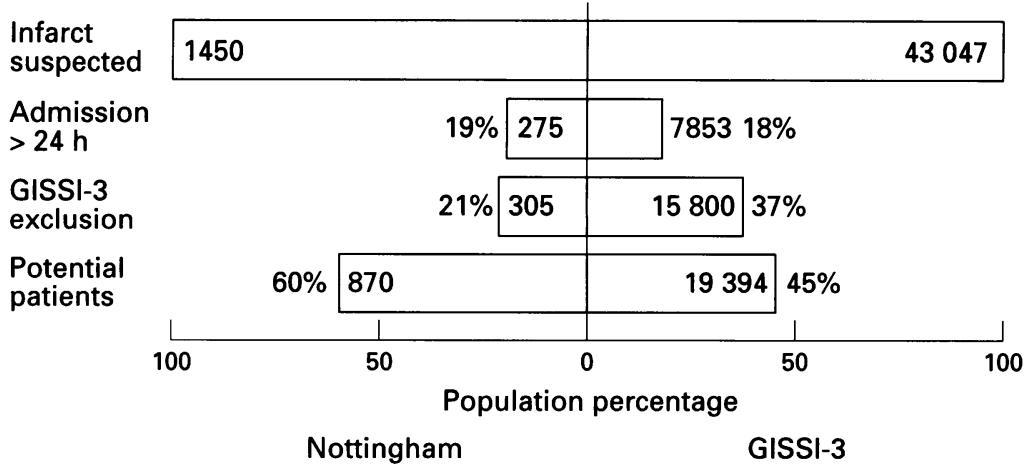

Figure 3 Comparison of Nottingham patients with those recruited in GISSI-3.

\begin{tabular}{|c|c|c|c|c|}
\hline $\begin{array}{l}\text { Infarct } \\
\text { suspected }\end{array}$ & \multicolumn{2}{|l|}{3208} & \multicolumn{2}{|l|}{$?$} \\
\hline $\begin{array}{l}\text { Admission } \\
>24 \mathrm{~h}\end{array}$ & \multicolumn{2}{|r|}{$12 \% 400$} & $?$ & \\
\hline $\begin{array}{l}\text { ISIS-4 } \\
\text { exclusion }\end{array}$ & \multicolumn{2}{|r|}{\begin{tabular}{ll|l}
$25 \%$ & 800
\end{tabular}} & $?$ & \\
\hline \multirow[t]{5}{*}{$\begin{array}{l}\text { Potential } \\
\text { patients }\end{array}$} & \multirow[t]{2}{*}{$63 \%$} & 2008 & 58000 & \\
\hline & & 1 & 1 & $\dashv$ \\
\hline & 100 & 50 & 50 & 100 \\
\hline & \multicolumn{4}{|c|}{ Population percentage } \\
\hline & & Jottingham & ISIS- & \\
\hline
\end{tabular}

Figure 4 Comparison of Nottingham patients with those recruited in ISIS-4.

with suspected infarcts over a two year period in Nottingham only $1768 \quad(33 \%$ of all patients) had confirmed infarcts; of these 335 ( $19 \%$ of those with infarcts) were excluded by an early death or ineligible age and a further 416 ( $24 \%$ of those with infarcts) excluded by specific SAVE criteria. If the prevalence of left ventricular dysfunction was similar in both groups of patients only 142 ( $8 \%$ of all those with infarcts) patients would have met the entry criteria for the SAVE study.

\section{COMPARISON OF NOTTINGHAM PATIENTS WITH} THOSE RECRUITED IN GISSI-3

GISSI-3 study population

The GISSI-3 study randomised patients with suspected myocardial infarction admitted to coronary care (in the presence of an abnormal electrocardiogram) to treatment with lisinopril within 24 hours of the onset of symptoms. Treatment was continued for 42 days. Figure 3 shows that 43047 patients were considered for study but 7853 (18\%) were excluded because of a delay in admission. A further $15800(37 \%)$ were excluded because of severe or pre-existing cardiac failure, contraindications to ACE inhibitor therapy, sustained hypotension, or because of clinical instability. This figure also includes 6670 patients who were not enrolled for administrative reasons. Overall 19394 patients (45\%) were randomised to treatment. Myocardial infarction was confirmed in $95 \%$ of these.

Nottingham patients

Applying the GISSI-3 criteria to the Nottingham patients (fig 3 ) shows that 1450 patients with suspected infarcts had an abnor- mal electrocardiogram when they were admitted to coronary care. $275(19 \%)$ of patients were admitted after 24 hours and a further $305(21 \%)$ would have been excluded in accordance with the GISSI-3 protocol. Overall $870(60 \%)$ of patients were eligible for treatment. Myocardial infarction was confirmed in $75 \%$ of these.

COMPARISON OF NOTTINGHAM PATIENTS WITH THOSE RECRUITED IN ISIS-4

The ISIS-4 study was deliberately designed to minimise the exclusion criteria seen in other studies and so all patients with suspected myocardial infarction admitted to coronary care within 24 hours of the onset of symptoms (with or without electrocardiographic changes) were considered. Over 58000 patients entered the study but the data regarding those excluded are not yet available. Myocardial infarction was confirmed in over $90 \%$. By the ISIS criteria 3208 patients in Nottingham would have been considered suitable but $400(12 \%)$ were admitted after 24 hours (fig 4). A further 800 (25\%) were excluded by the ISIS protocol, leaving 2008 $(63 \%)$ of those with suspected infarcts) patients for study. Myocardial infarction was confirmed in $72 \%$.

\section{ESTIMATED POTENTIAL NUMBER OF LIVES SAVED}

Figure 5 shows the estimated number of lives that might have been saved each year in Nottingham if patients had been given ACE inhibitors according to the AIRE, SAVE, GISSI-3, and ISIS-4 protocols.

In the AIRE study 57 lives per 1000 patients treated were saved, which would result in 20 lives saved per year in Nottingham. The mortality benefits were seen after 30 days and treatment of appropriate patients in Nottingham in the first 30 days would have prevented seven deaths. In the SAVE study 42 deaths per 1000 patients treated were prevented but because not many were eligible for treatment only three lives would have been saved in Nottingham. In the GISSI-3 trial eight lives per 1000 were saved and in the ISIS-4 study five lives per 1000 . These protocols would have prevented four and five deaths respectively each year in Nottingham.

\section{ESTIMATED COST OF ACE INHIBITOR} TREATMENT

Figure 5 also shows the total estimated drug cost of treating those with suspected or confirmed infarcts admitted each year in Nottingham with ACE inhibitors given according to the AIRE, SAVE, GISSI-3, and ISIS- 4 protocols. These data assume that all patients take the ACE inhibitor as per protocol and that none withdraw from medication. Treatment with ramipril would cost about $£ 110000$ because nearly $40 \%$ of those admitted with infarcts would be eligible for treatment for a mean of 15 months. Each life saved would cost approximately $£ 5400$. The treatment costs over the shorter period of 30 


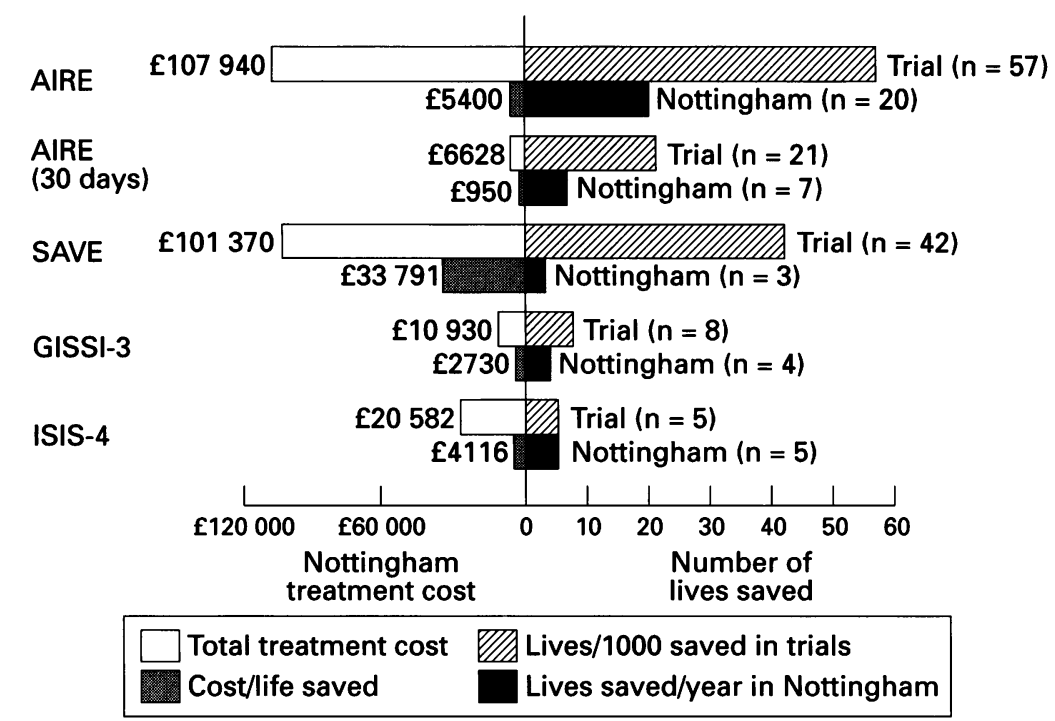

Figure 5 Cost benefit analysis of treating all infarcts admitted to Nottingham hospitals in one year according to major trial criteria.

days both in total and for each life saved, are much lower (fig 5). Total treatment costs with captopril as in the SAVE study would be slightly less because fewer patients would require treatment but the cost per life saved would be $£ 33791$ per patient. Patients randomised in GISSI-3 were only treated for 42 days and so total treatment costs would be low at $£ 10$ 930. The drug cost per life saved would be approximately $£ 2730$. Similarly patients in ISIS-4 were only treated for 28 days, giving a total treatment cost of $£ 20582$ and a cost per life saved of $£ 4116$.

\section{Discussion}

Ideally, good clinical practice should be based on the results of well conducted clinical trials. There may be difficulties in incorporating results from large-scale trials into clinical practice because the benefits observed in trials often reflect a strictly defined population and do not necessarily apply to a wider selection of patients.

All four recent trials confirm that there is benefit from treatment with an ACE inhibitor after acute myocardial infarction, with reductions in deaths, myocardial infarction, and progression to symptomatic heart failure. ${ }^{5-8}$ Because each trial recruited a different population of patients, it is important to establish how closely the trial populations approximated to the patients who are admitted to our hospitals.

Which trial is most appropriate for the types of patients admitted to the Nottingham hospitals with acute myocardial infarction? Nearly $40 \%$ of our patients with confirmed infarcts fit the AIRE study criteria. ${ }^{6}$ This reflects the high numbers of patients in the Nottingham population with clinical evidence of heart failure after admission. The reason for this is not clear because the numbers of patients receiving thrombolysis or with anterior infarcts were similar to those in the AIRE study. Fewer patients ( $7 \%$ of those with infarcts) in the AIRE study were eventually randomised to treatment but this also includes those patients in whom consent or long-term assessment was not possible. If the $40 \%$ of patients eligible in Nottingham were to receive the ACE inhibitor ramipril from around the fifth day after myocardial infarction (approximately 347 patients each year), we might expect to prevent 20 deaths in the next 15 months. The cost per life saved would be $£ 5520$. The cost benefit of the AIRE study seems even more remarkable when assessed at 30 days. This early effect on mortality was not described in the SAVE study, perhaps reflecting the different patient groups. The AIRE trial has, however, been criticised for failing to consider those patients with asymptomatic left ventricular dysfunction, who also have a high mortality after infarction. ${ }^{1011}$ The AIRE study would have excluded a significant proportion of Nottingham patients (32\% of those with confirmed infarcts) with no signs of heart failure, of whom $36 \%$ had anterior infarcts and nearly $50 \%$ were not treated with thrombolytic agents. Both these latter groups are thought to represent subgroups at particular risk of ventricular dysfunction and dilatation and these too may therefore benefit from ACE inhibition. ${ }^{12}$

Few of our patients matched the SAVE criteria, ${ }^{5}$ so few patients might expect to benefit from receiving an ACE inhibitor around 11 days after myocardial infarction. The number of lives saved is small and because treatment was continued for $\mathbf{4 2}$ months the drug cost of saving a life is high, over ten times higher than in the GISSI-3 population and over six times higher than in the AIRE study. Furthermore before administration of captopril, nearly 900 patients would require formal assessment of ejection fraction, which using echocardiography in Nottingham would cost approximately $£ 32000$. Another important feature of the SAVE study was the use of coronary angiography and exercise tests to investigate postinfarct ischaemia. Almost $60 \%$ of patients (equivalent to 420 each year in Nottingham) required cardiac catheterisation and nearly $25 \%$ proceeded to coronary bypass surgery or angioplasty before entering the study. Most district general hospitals would be unable to provide this service.

Sixty per cent of Nottingham patients with suspected myocardial infarction and abnormal electrocardiograms satisfied the GISSI-3 criteria. $^{7}$ If these patients had been treated with lisinopril within 24 hours of the onset of symptoms, 435 patients per year would have been treated and four deaths prevented. The cost per life saved is less because treatment was for 42 days only. The number of deaths prevented, however, is also low compared with the other studies. This might reflect the use of ACE inhibitors within 24 hours, as seen in the CONSENSUS II study ${ }^{13}$ or alternatively the short duration of follow up. In both AIRE and SAVE the survival curves diverged and further reductions in mortality emerged in the long term.

The effects on mortality seen in GISSI-3 
resembled the preliminary findings of the ISIS-4 trial. ${ }^{14}$ Patients recruited into ISIS-4 were randomised to treatment with captopril within 24 hours of the onset of symptoms, however (in contrast to GISSI-3), patients with a normal electrocardiogram were also included. This would result in a higher number of patients in Nottingham (1004 per year) eligible for treatment and so total treatment costs would be increased. Subgroup analysis of the preliminary ISIS data suggests that the benefits of ACE inhibitors are greater in patients taking diuretics or with confirmed anterior infarcts. ${ }^{14}$ Of the 1004 patients each year in Nottingham eligible for ISIS-4 $42 \%$ were taking diuretics and $32 \%$ had confirmed anterior infarcts.

The benefits of ACE inhibition after a myocardial infarct are not restricted to effects on mortality. Recurrent myocardial infarction and the progression and development of heart failure may also be reduced. This must also be considered in assessing the overall cost benefits of drug treatment. From the published data it was not possible to calculate the magnitude of these benefits in our patients for all four studies. Using the $\mathrm{AIRE}^{6}$ data as an example, however, in Nottingham we might expect to prevent 22 episodes of severe heart failure each year if patients were treated as specified in the original protocol. Assuming most of these patients would require admission this represents a potential cost saving of nearly $£ 40000$ each year. ${ }^{15}$

Despite differences in patient recruitment, the message from the clinical trials is clearsuitable patients should be offered treatment with an ACE inhibitor. Identification of some of these patients requires formal assessment of ventricular function, probably by echocardiography. Routine screening of all confirmed infarcts would significantly increase the annual workload of our echocardiography department but this demand could be reduced by targeting high risk groups as defined in the large scale trials. Our data show that had any of these multicentre trials been conducted in Nottingham in 1989 and 1990, $63 \%$ of all our patients with suspected myocardial infarction would have met at least one of the trials' criteria and so have been suitable for an ACE inhibitor. The GISSI-3 and ISIS-4 trial protocols were somewhat cheaper but the number of lives saved was small. In terms of current clinical practice the AIRE study is likely to have the greatest impact but we should be prepared to offer ACE inhibitors routinely to patients satisfying the criteria of any of the recent clinical trials.

1 Hammermeister KE, DeRouen TA, Dodge HT. Variables predictive of survival in patients with coronary heart disease: selection by univariate and multivariate analyses from the clinical, electrocardiographic, exercise, arteriofrom the clinical, electrocardiographic, exercise, arterioGraphic, and quantitative

2 Pfeffer MA, Pfeffer JM, Lamas GA. Development and prevention of congestive heart failure following myocardial infarction. Circulation 1993;87(suppl IV):120-5.

3 Kannel WB. Epidemiology and prevention of cardiac failure: Framingham Study insights. Eur Heart $\mathcal{F}$ 1987;suppl $\mathrm{F}: 23-6$.

4 Townend JN, Littler WA. Angiotensin converting enzyme inhibitors in heart failure: how good are they? Br Heart $\mathcal{f}$ 1993;69:373-5.

5 Pfeffer MA, Braunwald E, Moye LA, et al. Effect of captopril on mortality and morbidity in patients with left venpril on mortality and morbidity in patients with left venMed 1992;327:669-77.

6 The AIRE Study Investigators. Effect of ramipril on mortality and morbidity of survivors of acute myocardial infarction with clinical evidence of heart failure. Lance 1993;342:821-8.

7 GISSI-3 Investigators. GISSI-3: Effects of lisinopril and transdermal glyceryl trinitrate singly and together on 6 week mortality and ventricular function after acute myocardial infarction. Lancet 1994;343:1115-22.

8 ISIS-4 Collaborative Group, Oxford, UK. Fourth international study of infarct survival: Protocol for a large simple study of the effects of oral mononitrate, of oral captopril, study of the effects of oral mononitrate, or oral captopril, 1991;68:87D-100D.

9 Rowley JM, Mounser P, Harrison HA, Skene AM, Hampton JR. Management of myocardial infarction: implications for current policy derived from the Nottingham Heart Attack Register. Br Heart $f 1992$; 67:255-62.

10 McMurray J. ACE inhibitors after myocardial infarction Lancet 1993;342:1418.

11 Gottlieb S, Moss AJ, Mcdermot M, Eberley S. Inerrelation of left ventricular ejection fraction, pulmonary congestion and outcome in acute myocardial infarction. $A m \mathcal{F}$ Cardiol 1992;69:977-84.

12 Poole-Wilson PA. ACE inhibitors after myocardial infarction. Lancet 1994;343:289-90.

13 Swedberg K, Held P, Kjekshus J, et al. On behalf of the CONSENSUS II study group. Effect of early administration of enalapril on mortality in patients with acute myocardial infarction. Results of the Cooperative North myocardial infarction. Results of the Cooperative North Scandinavian Enalapril Survival Study
SUS II). N Engl f Med 1992;327:678-84.

14 ISIS-4 Collaborative Group. Fourth International Study of Infarct Survival: protocol for a large simple study of the effect of oral mononitrate, or oral captopril, and of intravenous magnesium. Am $\mathcal{F}$ Cardiol 1991;68(suppl) $87-100$

15 McMurray J, Hart W, Rhodes G. An evaluation of the cost of heart failure to the National Health Service in the UK Br $\mathcal{Y}$ Med Economics 1993;6:99-110. 